\begin{tabular}{|c|l|}
\hline Title & $\begin{array}{l}\text { ON GENERA EX ISTENCE RESULT S FOR ONE-DIMENSIONAL SINGULAR DIFFU SION EQUA TIONS WITH } \\
\text { SPATIALL INHOMOGENEOUS DRIVING FORCE }\end{array}$ \\
\hline Author(s) & Giga, Mi-Ho; GIGA, Y OSHIKAZU; Nakay asu, A tsushi \\
\hline Citation & Hokkaido University Preprint Series in Mathematics, 1032, 1-20 \\
\hline Issue Date & 2013-4-19 \\
\hline DOI & 10.14943/84176 \\
\hline Doc URL & http:/hdl.handle.net/2115/69836 \\
\hline Type & bulletin (article) \\
\hline
\end{tabular}

Instructions for use 


\title{
ON GENERAL EXISTENCE RESULTS FOR ONE-DIMENSIONAL SINGULAR DIFFUSION EQUATIONS WITH SPATIALLY INHOMOGENEOUS DRIVING FORCE
}

\author{
MI-HO GIGA, YOSHIKAZU GIGA, AND ATSUSHI NAKAYASU
}

\begin{abstract}
A general anisotropic curvature flow equation with singular interfacial energy and spatially inhomogeneous driving force is considered for a curve given by the graph of a periodic function. We prove that the initial value problem admits a unique global-in-time viscosity solution for a general periodic continuous initial datum. The notion of a viscosity solution used here is the same as proposed by Giga, Giga and Rybka, who established a comparison principle. We construct the global-in-time solution by careful adaptation of Perron's method.
\end{abstract}

\section{INTRODUCTION}

In this paper we study a one-dimensional nonlinear degenerate parabolic equation whose diffusion effect is very strong at particular slopes of unknown functions. We are in particular interested in an equation, where the driving force term is spatially inhomogeneous. A typical example is a quasilinear equation

$$
u_{t}=a\left(u_{x}\right)\left[\left(W^{\prime}\left(u_{x}\right)\right)_{x}+\sigma(t, x)\right],
$$

where $W$ is a given convex function on $\mathbf{R}$ but may not be of class $C^{1}(\mathbf{R})$ so that its derivative $W^{\prime}$ may have jump discontinuities and $\sigma$ is a given Lipschitz function depending on the space variable $x$ as well as the time variable $t$; here $a$ is a given nonnegative continuous function, and $u_{t}$ and $u_{x}$ denote the time and the space derivative of an unknown function $u=u(t, x)$.

In order to explain the motivation of this work, let us consider an evolution law of a curve $\Gamma_{t} \subset \mathbf{R}^{2}$ moved by an anisotropic curvature flow

$$
V=M_{0}(\mathbf{n})\left(\kappa_{\gamma_{0}}+\sigma\right) \text { on } \Gamma_{t},
$$

where $V$ is the normal velocity of the evolving curve in the direction of the normal vector $\mathbf{n}$ and let the mobility $M_{0}$ and the surface energy density $\gamma_{0}$ be positive functions on the unit circle; the term $\kappa_{\gamma_{0}}$ called a nonlocal curvature is the first variation of surface energy. We note that if $\gamma_{0}$ is the constant 1 , then $\kappa_{\gamma_{0}}$ is nothing but usual curvature $\kappa$; the quantity $\kappa_{\gamma_{0}}$ formally equals $\left(\left(\gamma_{0}\right)_{\theta \theta}+\gamma_{0}\right) \kappa$ if one writes $\gamma_{0}$ as a function of the argument $\theta$ of $\mathbf{n}=(\cos \theta, \sin \theta)$. The equation (D2) appears in crystal growth as an equation to describe the interface of two phases; see, e.g., [2]. 


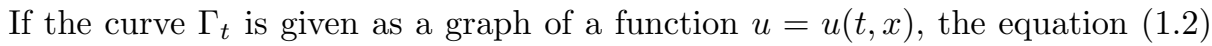
then becomes of the form $(\mathbb{\mathbb { L }} \mathbb{\mathbb { D }})$ with

$$
\begin{aligned}
a(p) & =M(p,-1), & M(p, q) & =\sqrt{p^{2}+q^{2}} M_{0}\left(\frac{(p, q)}{\sqrt{p^{2}+q^{2}}}\right), \\
W(p) & =\gamma(p,-1), & \gamma(p, q) & =\sqrt{p^{2}+q^{2}} \gamma_{0}\left(\frac{(p, q)}{\sqrt{p^{2}+q^{2}}}\right) .
\end{aligned}
$$

Assume that the Frank diagram $F:=\left\{(p, q) \in \mathbf{R}^{2} \mid \gamma(p, q) \leq 1\right\}$ is convex so that $W$ is a convex function. If $F$ has a smooth $\left(C^{2}\right)$ boundary $\partial F$, the theory of (ए.2) is well developed [G], [9], [16]. Indeed, since $W$ is $C^{2}(\mathbf{R})$, we are able to apply the classical theory of viscosity solutions [ [ [] to the equation (ㅁ. D). We are concerned with the case that $\partial F$ is of class $C^{2}$ except finitely many points. A typical example of $F$ is a polygon so that $W$ is a piecewise linear function. For examples if $\gamma$ is a crystalline energy of the form

$$
\gamma(p, q)=|p|+|q|,
$$

then $W^{\prime \prime}(p)$ is twice the Dirac delta function $\delta$ and so the equation (ㅁ. $)$ formally becomes

$$
u_{t}=a\left(u_{x}\right)\left[2 \delta\left(u_{x}\right) u_{x x}+\sigma\right],
$$

which is not a classical partial differential equation.

Admissible curves such as polygons moving by a crystalline energy with no driving force have been studied by Taylor [1], 201] and by Angenent and Gurtin [T]. For the evolution law of graphs $(\mathbb{\square} . \mathbb{C})$ a notion of solutions is introduced by adapting the subdifferential theory [II0] $(\sigma=0)$ and [II]. Elliott, Gardiner and Schätzle [8] study relationship between the solutions in the sense of [II0] and admissible curves. When $\sigma$ is independent of $x$, the theory of viscosity solutions to (ㅁ. $)$ and (․ㄹ) is established in a series of papers [12], [13], [14].

The goal of this paper is to establish a global-in-time existence theorem of a viscosity solution for a class of equations including $(\mathbb{\square}, \mathbb{D})$ with a given continuous periodic initial condition. Our result is a generalization of [12, Section 8, 9] to the equation with spatially inhomogeneous driving force. Notion of viscosity solutions to $(\mathbb{L}$. $)$ with $\sigma$ depending on $x$ is introduced in [15], where a comparison theorem is established. The authors of [15] also show some existence results by showing that a special semi-explicit variational solution studied in [I7] is a viscosity solution but their initial data is very restrictive. We also point out that in a recent paper by

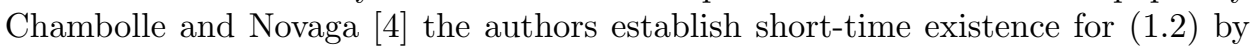
time-discrete implicit scheme, which is introduced in [5], [3]. Our argument based on the theory of viscosity solutions is completely different from theirs and can be applied to a fully nonlinear equation.

Following [15], let us consider an energy functional which formally equals

$$
\Phi[f]=\int_{\mathbf{T}}\left(W\left(f_{x}\right)-\sigma f\right) d x
$$

for a smooth function $f$; we assumed a periodic boundary condition so that $\mathbf{T}=$ $\mathbf{R} / \omega \mathbf{Z}$ with $\omega>0$. Let $\partial^{0} \Phi[f]$ be the canonical restriction of the subdifferential $\partial \Phi[f]$ in the Hilbert space $H:=L^{2}(\mathbf{T})$, i.e.

$$
\partial^{0} \Phi[f]=\arg \min \left\{\|\lambda\|_{H} \mid \lambda \in \partial \Phi[f]\right\} .
$$


As mentioned in [П]], the above minimizing problem is equivalent to an obstacle problem: The condition $\lambda \in-\partial \Phi[f]$ holds if and only if $\lambda$ is of the form $\lambda=\xi^{\prime}$ such that $\xi \in \partial W\left(f_{x}\right)+Z$ a.e. on $\mathbf{T}$, where $Z$ is a primitive function of $\sigma$, i.e. $Z_{x}=\sigma$. Therefore, we minimize

$$
\left\{\int_{\mathbf{T}}\left|\xi^{\prime}\right|^{2} d x \mid \xi \in \partial W\left(f_{x}\right)+Z \text { a.e. on } \mathbf{T}\right\} .
$$

There might be a chance that there is no such $\xi$ satisfying $\xi \in \partial W\left(f_{x}\right)+Z$ a.e. on $\mathbf{T}$. We need to require special structure to guarantee the existence of such $\xi$. A sufficient condition is that $f$ is flat (facet) on a nontrivial interval (called a faceted region) containing each fixed point $x$ whenever $\partial W$ has a jump at $f_{x}(x)$. Such a function $f$ is called a faceted function and we see that $(\mathbb{L} .3)$ admits a unique minimizer $\bar{\xi}$ for a faceted function $f$ since the problem is convex. It is natural to guess that $\bar{\xi}^{\prime}$ gives a candidate for the value of the nonlocal curvature

$$
\Lambda_{W}^{\sigma}(f)(x)=\left(W^{\prime}\left(f_{x}\right)\right)_{x}+\sigma(x) .
$$

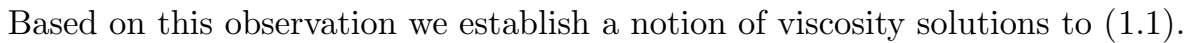

We prove the existence theorem by Perron's method, which is standard in the theory of viscosity solutions for regular equations; we refer the reader to [iz], [ [ $]$. In our problem, however, it is necessary to modify a smooth faceted test function keeping its property. In the previous work [I:2] it suffices to modify the test function outside the faceted region. However, this method heavily relies on the fact that the nonlocal curvature $\Lambda_{W}^{\sigma}(f)$ is constant on a faceted region when $\sigma$ is independent of $x$.

The main idea to solve this problem is to find a small effective region which determines the quantity of the nonlocal curvature. We construct a modification as in the previous work [12] using the effective region instead of the faceted region. Then the argument works well for our setting with the spatially inhomogeneous driving force term $\sigma$.

This paper is organized as follows. In Section $\nabla$ we recall the definition of faceted functions and the nonlocal curvature $\Lambda_{W}^{\sigma}$ and define generalized solutions for the equations. In Section [3 we describe how to construct an effective region and modifications for test functions. In Section $⿴$ we prove Perron type existence theorems and Section 15 is devoted to proving the existence theorem for periodic initial data.

\section{Definitions OF GENERALized SOlutions}

In this section we recall some notions of functions and the nonlocal curvature $\Lambda_{W}^{\sigma}$ introduced in [15, Section 2] and define generalized solutions for fully nonlinear equations of the form

$$
u_{t}+F\left(t, u_{x}, \Lambda_{W}^{\sigma}(u)\right)=0 \quad \text { in } \mathcal{Q}:=(0, T) \times \Omega,
$$

where $T>0$ and $\Omega$ is an open set in $\mathbf{R}$. We assume the following conditions throughout this paper.

(W) Assume $W$ is a convex function on $\mathbf{R}$ with values in $\mathbf{R}$ of class $C^{2}$ outside a closed discrete set $P$ and that its second derivative $W^{\prime \prime}$ is bounded in any compact set except all points in $P$.

(S) The continuous function $\sigma=\sigma(t, x)$ on $[0, T] \times \Omega$ is Lipschitz continuous in $x$ uniformly with respect to $t$, i.e. there exists a constant $L$ such that

$$
|\sigma(t, x)-\sigma(t, y)| \leq L|x-y| \quad \text { for all } t \in[0, T), x, y \in \Omega \text {. }
$$


(F1) $F$ is continuous on $[0, T] \times \mathbf{R} \times \mathbf{R}$ with values in $\mathbf{R}$.

(F2) $F(t, p, X) \leq F(t, p, Y)$ for all $t \in[0, T], p \in \mathbf{R}, X \geq Y$.

The discrete set $P$ in $(\mathrm{W})$ is either a finite set or a countable set having no accumulation point in $\mathbf{R}$. If $P$ is nonempty, $P$ is of form $\left\{p_{j}\right\}_{j=1}^{m},\left\{p_{j}\right\}_{j=1}^{\infty},\left\{p_{j}\right\}_{j=-1}^{-\infty}$ or $\left\{p_{j}\right\}_{j=-\infty}^{\infty}$, where $\left\{p_{j}\right\}$ is a strictly increasing sequence $p_{j}<p_{j+1}$ with $\lim _{j \rightarrow \infty} p_{j}=$ $\infty$ and $\lim _{j \rightarrow-\infty} p_{j}=-\infty$, and $m$ is a positive integer. We often let $\sigma(t)$ denote the function $\sigma(t)(x)=\sigma(t, x)$ for $t \in[0, T)$. We say that a family of a functions $\sigma_{t}$ on $\Omega$ is equi-Lipschitz continuous if there exists a constant $L$ such that $\left|\sigma_{t}(x)-\sigma_{t}(y)\right| \leq L|x-y|$ for all $t$ and $x, y \in \Omega$. Our assumption (S) is equivalent to saying that $\sigma(t)$ on $\Omega$ is equi-Lipschitz continuous.

2.1. Faceted functions. We first define a notion of a faceted function.

Definition 2.1 (Faceted function). A function $f \in C^{1}(\Omega)$ is faceted at a point $\hat{x} \in \Omega$ with slope $p \in \mathbf{R}$ (or $p$-faceted at $\hat{x}$ ) if there exists a closed nontrivial finite interval $I=\left[c_{l}, c_{r}\right] \subset \Omega$ containing $\hat{x}$ (i.e. $c_{l}, c_{r} \in \Omega$ satisfy $c_{l}<c_{r}$ and $c_{l} \leq \hat{x} \leq c_{r}$ ) such that

$$
\begin{array}{ll}
f^{\prime}(x)=p & \text { for all } x \in I, \\
f^{\prime}(x) \neq p & \text { for all } x \in J \backslash I
\end{array}
$$

with some neighborhood $J=\left(b_{l}, b_{r}\right) \subset \Omega$ of $I$. The closed interval $I$ is called a faceted region of $f$ containing $\hat{x}$. We say that a function $f$ is $P$-faceted at $\hat{x}$ if $f$ is $p$-faceted at $\hat{x}$ for some $p \in P$ and let

$$
C_{P}^{2}(\Omega):=\left\{f \in C^{2}(\Omega) \mid f \text { is } P \text {-faceted at } \hat{x} \text { whenever } f^{\prime}(\hat{x}) \in P\right\} .
$$

We also define the left transition number $\chi_{l}=\chi_{l}(f, \hat{x})$ and the right transition number $\chi_{r}=\chi_{r}(f, \hat{x})$ for a $p$-faceted function $f$ at $\hat{x}$ by

$$
\begin{aligned}
& \chi_{l}= \begin{cases}+1 & \text { if } f^{\prime}<p \text { on }\left(b_{l}, c_{l}\right), \\
-1 & \text { if } f^{\prime}>p \text { on }\left(b_{l}, c_{l}\right),\end{cases} \\
& \chi_{r}= \begin{cases}+1 & \text { if } f^{\prime}>p \text { on }\left(c_{r}, b_{r}\right), \\
-1 & \text { if } f^{\prime}<p \text { on }\left(c_{r}, b_{r}\right)\end{cases}
\end{aligned}
$$

Let $R(f, \hat{x})=\left[c_{l}, c_{r}\right]$ denote a maximal closed interval containing $\hat{x}$ on which $f^{\prime}$ is constant, i.e.

$$
\begin{aligned}
& c_{l}:=\inf \left\{x \in \Omega \mid f^{\prime}(y)=f^{\prime}(\hat{x}) \text { for all } y \in[x, \hat{x}]\right\}, \\
& c_{r}:=\sup \left\{x \in \Omega \mid f^{\prime}(y)=f^{\prime}(\hat{x}) \text { for all } y \in[\hat{x}, x]\right\} .
\end{aligned}
$$

The interval $R(f, \hat{x})$ is nothing but the faceted region if $f$ is a $P$-faceted function at $\hat{x}$.

Remark 2.2. We note that a $p$-faceted function $f$ at $\hat{x}$ agrees with an affine function

$$
\ell_{p}(x):=p(x-\hat{x})+f(\hat{x})
$$


on $I=R(f, \hat{x})$ and that

$$
\begin{aligned}
& \chi_{l}= \begin{cases}+1 & \text { if } f>\ell_{p} \text { on }\left(b_{l}, c_{l}\right), \\
-1 & \text { if } f<\ell_{p} \text { on }\left(b_{l}, c_{l}\right),\end{cases} \\
& \chi_{r}= \begin{cases}+1 & \text { if } f>\ell_{p} \text { on }\left(c_{r}, b_{r}\right), \\
-1 & \text { if } f<\ell_{p} \text { on }\left(c_{r}, b_{r}\right) .\end{cases}
\end{aligned}
$$

2.2. Nonlocal curvature with a nonuniform driving force. We next recall the definition of the nonlocal curvature for a smooth faceted function. Assume (W) and that

$\sigma$ is a Lipschitz function on $\Omega$.

For $f \in C_{P}^{2}(\Omega)$ and $\hat{x} \in \Omega$ define the nonlocal curvature $\Lambda_{W}^{\sigma}(f)(\hat{x})$ as below.

On one hand, if $f^{\prime}(\hat{x}) \notin P$, we set

$$
\Lambda_{W}^{\sigma}(f)(\hat{x})=W^{\prime \prime}\left(f^{\prime}(\hat{x})\right) f^{\prime \prime}(\hat{x})+\sigma(\hat{x})
$$

as expected. On the other hand, if $p:=f^{\prime}(\hat{x}) \in P$, i.e. $f$ is $p$-faceted at $\hat{x}$, the definition is more involved since it is based on the obstacle problem (ㄸ.3).

Let $Z$ be a primitive function of $\sigma$ and let

$$
\Delta=|\partial W(p)|=\lim _{q \downarrow p} W^{\prime}(q)-\lim _{q \uparrow p} W^{\prime}(q) .
$$

We also take the faceted region $I=R(f, \hat{x})=\left[c_{l}, c_{r}\right]$ and the transition numbers $\chi_{l}=\chi_{l}(f, \hat{x}), \chi_{r}=\chi_{r}(f, \hat{x})$. We note that

(2.3) $Z \in C^{1,1}(I), \Delta>0, I$ is a nontrivial closed interval and $\chi_{l}, \chi_{r} \in[-1,1]$.

For later convenience we have defined $K$ for $\chi_{l}, \chi_{r}$ whose values are in $[-1,1]$ not necessarily in $\{ \pm 1\}$. Let $K=K_{\chi_{l} \chi_{r}}^{Z, \Delta, I}$ be the set of all $\xi \in H^{1}(I)$ satisfying an obstacle condition

$$
Z(x)-\Delta / 2 \leq \xi(x) \leq Z(x)+\Delta / 2 \text { for all } x \in I
$$

and a boundary condition

$$
\xi\left(c_{l}\right)=Z\left(c_{l}\right)-\chi_{l} \Delta / 2, \quad \xi\left(c_{r}\right)=Z\left(c_{r}\right)+\chi_{r} \Delta / 2 .
$$

We now consider the functional $J=J_{\chi_{l} \chi_{r}}^{Z, \Delta, I}$ on $L^{2}(I)$ defined by

$$
J[\xi]= \begin{cases}\int_{I}\left|\xi^{\prime}(x)\right|^{2} d x & \text { if } \xi \in K, \\ \infty & \text { otherwise. }\end{cases}
$$

It is easy to see that $K$ is a closed convex set with respect to $H^{1}$ norm and thus $J$ admits a unique minimizer denoted by $\bar{\xi}=\xi_{\chi_{l} \chi_{r}, \Delta}^{Z, I}$.

An equivalent condition to being a minimizer of the obstacle problem is known. Assume (ए.3). For $\xi \in K$ define the upper coincidence set $D_{+}$and the lower coincidence set $D_{-}$by

$$
D_{ \pm}=D_{ \pm}(\xi)=\{x \in I \mid \xi(x)=Z(x) \pm \Delta / 2\} .
$$

We say that $\xi$ satisfies concave-convex condition if $\xi$ is concave outside the upper coincidence set $D_{+}$and convex outside the lower coincidence set $D_{-}$.

Proposition 2.3 (Characterization of minimizer). A function $\xi \in K$ is the minimizer of $J$ if and only if $\xi$ satisfies the concave-convex condition. 
This proposition is proved in the same way as in [15, Proposition 2.2], which shows the equivalence with the assumption $\chi_{l}, \chi_{r}= \pm 1$, and so we omit it. Noting that Proposition [2.3 in particular implies that the minimizer of the obstacle problem $\bar{\xi}$ belongs to $C^{1,1}(I)$, so we define

$$
\Lambda_{\chi_{l} \chi_{r}}^{Z^{\prime}}(x ; I, \Delta)=\bar{\xi}^{\prime}(x) \text { for } x \in I .
$$

The reason we write $Z^{\prime}$ instead of $Z$ is that the derivative $\bar{\xi}^{\prime}$ depends on $Z$ only through its derivative. Proposition 2.3 also shows that restriction of $\bar{\xi}$ is also a minimizer of an obstacle problem on the restricted domain:

Corollary 2.4. Let $M=\left[c_{l}, c_{r}\right] \subset I$ be a nontrivial closed interval. Then,

$$
\xi_{\chi_{l} \chi_{r}}^{Z, I}=\xi_{\chi_{l}^{\prime} \chi_{r}^{\prime}}^{Z, \Delta, M} \quad \text { on } M
$$

with

$$
\chi_{l}^{\prime}=2\left(\bar{\xi}\left(c_{l}\right)-Z\left(c_{l}\right)\right) / \Delta, \quad \chi_{r}^{\prime}=2\left(\bar{\xi}\left(c_{r}\right)-Z\left(c_{r}\right)\right) / \Delta .
$$

Definition 2.5 (Nonlocal curvature). Assume (W) and (ए2). Let $f \in C_{P}^{2}(\Omega)$ and $\hat{x} \in \Omega$.

(i) If $f^{\prime}(\hat{x}) \notin P$, then define

$$
\Lambda_{W}^{\sigma}(f)(\hat{x})=W^{\prime \prime}\left(f^{\prime}(\hat{x})\right) f^{\prime \prime}(\hat{x})+\sigma(\hat{x}) .
$$

(ii) If $f$ is $P$-faceted at $\hat{x}$, then define

$$
\Lambda_{W}^{\sigma}(f)(\hat{x})=\Lambda_{\chi_{l} \chi_{r}}^{\sigma}(\hat{x} ; I, \Delta)
$$

with $\Delta=|\partial W(p)|, I=R(f, \hat{x}), \chi_{l}=\chi_{l}(f, \hat{x}), \chi_{r}=\chi_{r}(f, \hat{x})$.

We prepare several propositions on the nonlocal curvature.

Proposition 2.6 (Comparison). Assume $(W)$ and (区.2). Let $f, g \in C_{P}^{2}(\Omega)$ and $\hat{x} \in \Omega$. If $\max _{\Omega}(f-g)=(f-g)(\hat{x})$, then

$$
\Lambda_{W}^{\sigma}(f)(\hat{x}) \leq \Lambda_{W}^{\sigma}(g)(\hat{x}) .
$$

Proposition 2.7 (Continuity with respect to $\sigma$ and $x$ ). Assume $(W)$ and let $f \in$ $C_{P}^{2}(\Omega)$ and $\hat{x} \in \Omega$. Let $y, y_{k} \in R(f, \hat{x})$ and equi-Lipschitz continuous functions $\sigma$, $\sigma_{k}$ on $\Omega$ satisfy $y_{k} \rightarrow y$ and $\sigma_{k} \rightarrow \sigma$ uniformly. Then

$$
\Lambda_{W}^{\sigma_{k}}(f)\left(y_{k}\right) \rightarrow \Lambda_{W}^{\sigma}(f)(y) .
$$

Proposition 2.8 (Continuity with respect to $I$ ). Assume (ए2), $\chi_{l}, \chi_{r}= \pm 1$, $\Delta>0$. Let nontrivial intervals $I=\left[c_{l}, c_{r}\right], I^{k}=\left[c_{l}^{k}, c_{r}^{k}\right]$ of $\Omega$ satisfy $I^{k} \rightarrow I$, i.e. $c_{l}^{k} \rightarrow c_{l}$ and $c_{r}^{k} \rightarrow c_{r}$, and let $y \in I, y_{k} \in I_{k}$ satisfy $y_{k} \rightarrow y$. Then

$$
\Lambda_{\chi_{l} \chi_{r}}^{\sigma}\left(y_{k} ; I_{k}, \Delta\right) \rightarrow \Lambda_{\chi_{l} \chi_{r}}^{\sigma}(y ; I, \Delta) \text {. }
$$

Proposition [2.6] $[2.8$ are immediate consequence of [15, Theorem 2.8, 2.9, 2.12].

2.3. Admissible functions and definition of a generalized solution. We recall a natural class of test function.

Definition 2.9 (Admissible function). Let $I$ and $J$ be open intervals in $\mathbf{R}$. An admissible function on $\mathcal{Q}:=J \times I$ is a function $\varphi$ of the form

$$
\varphi(t, x)=f(x)+g(t) \quad \text { on } \mathcal{Q}
$$

with some functions $f \in C_{P}^{2}(I)$ and $g \in C^{1}(J)$. Let $A_{P}(\mathcal{Q})$ be the set of all admissible functions on $\mathcal{Q}$. 
We are now able to define a generalized solution in the viscosity sense for the singular parabolic equation (एत्]). For a real-valued function $u$ recall the upper semicontinuous envelope and the lower semicontinuous envelope

$$
\begin{aligned}
& u^{*}(t, x):=\lim _{\varepsilon \downarrow 0} \sup \{u(s, y)|(s, y) \in \mathcal{Q},| s-t|+| y-x \mid<\varepsilon\}, \\
& u_{*}(t, x):=\lim _{\varepsilon \downarrow 0} \inf \{u(s, y)|(s, y) \in \mathcal{Q},| s-t|+| y-x \mid<\varepsilon\}
\end{aligned}
$$

for $(t, x) \in \overline{\mathcal{Q}}$.

Definition 2.10 (Viscosity solution). A real-valued function $u$ on $\mathcal{Q}$ is a viscosity subsolution of (区一) in $\mathcal{Q}$ if $u^{*}<\infty$ in $[0, T) \times \bar{\Omega}$ and

$$
\varphi_{t}(\hat{t}, \hat{x})+F\left(\hat{t}, \varphi_{x}(\hat{t}, \hat{x}), \Lambda_{W}^{\sigma(\hat{t})}(\varphi(\hat{t}, \cdot))(\hat{x})\right) \leq 0
$$

whenever $(\hat{t}, \hat{x}) \in \mathcal{Q}$ and $\varphi \in A_{P}(\mathcal{Q})$ satisfy

$$
\max _{\mathcal{Q}}\left(u^{*}-\varphi\right)=\left(u^{*}-\varphi\right)(\hat{t}, \hat{x})
$$

A real-valued function $u$ on $\mathcal{Q}$ is a viscosity supersolution of (․]) in $\mathcal{Q}$ if $u_{*}>-\infty$ in $[0, T) \times \bar{\Omega}$ and

$$
\varphi_{t}(\hat{t}, \hat{x})+F\left(\hat{t}, \varphi_{x}(\hat{t}, \hat{x}), \Lambda_{W}^{\sigma(\hat{t})}(\varphi(\hat{t}, \cdot))(\hat{x})\right) \geq 0
$$

whenever $(\hat{t}, \hat{x}) \in \mathcal{Q}$ and $\varphi \in A_{P}(\mathcal{Q})$ satisfy

$$
\min _{\mathcal{Q}}\left(u_{*}-\varphi\right)=\left(u_{*}-\varphi\right)(\hat{t}, \hat{x})
$$

If $u$ is both a subsolution and a supersolution, $u$ is called a viscosity solution.

Hereafter we suppress the word "viscosity". A function $\varphi$ satisfying (2.6]) or (2.8) is called a test function of $u$ at $(\hat{t}, \hat{x})$.

The following propositions are easily derived.

Proposition 2.11 (Smooth solution and viscosity solution). We assume $(W),(S)$, (F2). If $\varphi \in A_{P}(\mathcal{Q})$ of the form (2.4) with $f \in C_{P}^{2}(\Omega)$ and $g \in C^{1}(0, T)$ satisfies (ए.5) (resp. (ए.]) for each $(\hat{t}, \hat{x}) \in \mathcal{Q}$, then $\varphi$ is a subsolution (resp. supersolution) of $(\underline{2 .})$ in $\mathcal{Q}$.

Proof. We only show that $\varphi$ is a subsolution. Fix $\psi \in A_{P}(\mathcal{Q})$ of the form

$$
\psi(t, x)=\tilde{f}(x)+\tilde{g}(t) \quad \text { on } \mathcal{Q}
$$

with $\tilde{f} \in C_{P}^{2}(\Omega)$ and $\tilde{g} \in C^{1}(0, T)$, and suppose that

$$
\varphi(t, x)-\psi(t, x)=f(x)-\tilde{f}(x)+g(t)-\tilde{g}(t)
$$

attains a maximum at a point $(\hat{x}, \hat{t}) \in \mathcal{Q}$. We then see that $f^{\prime}(\hat{x})=\tilde{f}^{\prime}(\hat{x})$ and $g^{\prime}(\hat{t})=\tilde{g}^{\prime}(\hat{t})$. Moreover, Proposition [2.6] yields

$$
\Lambda_{W}^{\sigma(\hat{t})}(f)(\hat{x}) \leq \Lambda_{W}^{\sigma(\hat{t})}(\tilde{f})(\hat{x}) .
$$

Therefore, we have

$$
\tilde{g}^{\prime}(\hat{t})+F\left(\hat{t}, \tilde{f}^{\prime}(\hat{x}), \Lambda_{W}^{\sigma(\hat{t})}(\tilde{f})(\hat{x})\right) \leq g^{\prime}(\hat{t})+F\left(\hat{t}, f^{\prime}(\hat{x}), \Lambda_{W}^{\sigma(\hat{t})}(f)(\hat{x})\right) \leq 0
$$

by (F2) and ([.5). 
Proposition 2.12 (Addition by affine functions). Let $u$ be a subsolution (resp. supersolution) of ([D]) in $\mathcal{Q}$ and $a, b \in \mathbf{R}$. Then $v(t, x)=u(t, x)-a x-b$ is $a$ subsolution (resp. supersolution) of

$$
v_{t}+F\left(t, v_{x}+a, \Lambda_{W_{a}}^{\sigma}(v)\right)=0 \text { in } \mathcal{Q},
$$

where $W_{a}(p)=W(p+a)$.

In order to show the existence of a solution by Perron's method we define a local version of the notion of solutions. We say that a function $\varphi \in C(\mathcal{Q})$ is locally admissible at a point $(\hat{t}, \hat{x}) \in \mathcal{Q}$ if $\varphi$ is admissible on $J \times I$ with some bounded open intervals $I$ and $J$ such that $\hat{t} \in J \subset(0, T)$ and $\hat{x} \in I \subset \Omega$.

Definition 2.13. A real-valued function $u$ on $\mathcal{Q}$ is a subsolution in the local sense of $(\mathbb{E . D})$ in $\mathcal{Q}$ if $u^{*}<\infty$ in $[0, T) \times \bar{\Omega}$ and (区.5) holds for all locally admissible $\varphi \in C(\mathcal{Q})$ at $(\hat{t}, \hat{x}) \in \mathcal{Q}$ satisfying (区ש) . A supersolution in the local sense is

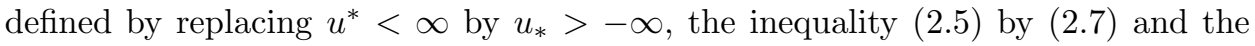
equality (2.6) by $([2.8)$ as before.

Lemma 2.14. A real-valued function $u$ on $\mathcal{Q}$ is a subsolution (resp. supersolution) of (区. $\mathbb{E})$ in $\mathcal{Q}$ if and only if $u$ is a subsolution (resp. supersolution) in the local sense of $($ 2.

These facts can be shown by the same argument as in [12, Section 6].

\section{EfFECTIVE REgION AND CANONICAL MODIFICATION}

In this section we construct an upper and lower modification $f^{\#, \varepsilon}$ and $f_{\#, \varepsilon}$ for a faceted function $f$ and a small number $\varepsilon>0$. These modifications play an important role in order to prove a Perron type existence theorem in the next section.

Definition 3.1. Let $f \in C(\Omega) \cap C_{P}^{2}\left(\Omega_{1}\right)$ satisfy $f^{\prime}(\hat{x})=0$ with an open interval $\Omega_{1}=\left(a_{l}, a_{r}\right) \subset \Omega$ and $\hat{x} \in \Omega_{1}$. Let

$$
\begin{aligned}
& p_{1}=\sup \{p \in P \cup\{-\infty\} \mid p<0\} \in[-\infty, 0), \\
& p_{2}=\inf \{p \in P \cup\{\infty\} \mid p>0\} \in(0, \infty] .
\end{aligned}
$$

Consider the case (i) $f^{\prime}(\hat{x})=0 \notin P$. We then define $M=\left[d_{l}, d_{r}\right]$ by

$$
d_{l}=d_{r}=\hat{x}, \quad \text { i.e. } M=\{\hat{x}\}
$$

and set

$$
f^{\#, \varepsilon}(x)=f^{\#}(x)=f(x)+(x-\hat{x})^{4} \quad \text { for } x \in \Omega .
$$

Let us note that there exists an open neighborhood $\Omega_{2}=\left(b_{l}, b_{r}\right) \subset \Omega_{1}$ of $\hat{x}$ such that

$$
\begin{gathered}
\frac{p_{1}}{2}<f^{\prime}(x)<\frac{p_{2}}{2} \quad \text { for all } x \in \Omega_{2}, \\
d_{l}+\frac{\sqrt[3]{p_{1}}}{2} \leq b_{l}<d_{l}, \quad d_{r}<b_{r} \leq d_{r}+\frac{\sqrt[3]{p_{2}}}{2} .
\end{gathered}
$$

Consider the case (ii) $f^{\prime}(\hat{x})=0 \in P$, i.e. $f$ is $P$-faceted at $\hat{x}$. Take the faceted region $\left[c_{l}, c_{r}\right]=R(f, \hat{x})$ and the minimizer of the obstacle problem $\xi$. Define $M=$ $\left[d_{l}, d_{r}\right]$ by

$$
\begin{aligned}
d_{l} & =\max \left\{x \leq \hat{x} \mid x \in D_{-}(\xi) \cup\left\{c_{l}\right\}\right\}, \\
d_{r} & =\min \left\{x \geq \hat{x} \mid x \in D_{+}(\xi) \cup\left\{c_{r}\right\}\right\} .
\end{aligned}
$$


Take an open interval $\Omega_{2}=\left(b_{l}, b_{r}\right) \subset \Omega_{1} \cap J$ such that (B.D) and (B.2) hold, where $J$ is the neighborhood of $R(f, \hat{x})$ appearing in Definition [2]. Define $f^{\#, \varepsilon}$ for each $\varepsilon>0$ as below: First set

$$
f^{\#, \varepsilon}(x)=f(x)=f(\hat{x}) \quad \text { for } x \in M=\left[d_{l}, d_{r}\right] .
$$

If $d_{l} \in D_{-}(\xi)$, set

$$
f^{\#, \varepsilon}(x)=f(x)+\left(x-d_{l}\right)^{4} \quad \text { for } x \in \Omega, x \leq d_{l} .
$$

If $d_{l} \notin D_{-}(\xi)$, that is $d_{l}=c_{l}$ and $d_{l} \in D_{+}(\xi)$, set

$$
f^{\#, \varepsilon}(x)= \begin{cases}f\left(d_{l}\right)=f(\hat{x}) & \text { for } x \in\left[d_{l}-\varepsilon, d_{l}\right], \\ f(x+\varepsilon) & \text { for } x \in\left[b_{l}, d_{l}-\varepsilon\right], \\ f(x)+f\left(b_{l}+\varepsilon\right)-f\left(b_{l}\right) & \text { for } x \in \Omega, x \leq b_{l} .\end{cases}
$$

If $d_{r} \in D_{+}(\xi)$, set

$$
f^{\#, \varepsilon}(x)=f(x)+\left(x-d_{r}\right)^{4} \quad \text { for } x \in \Omega, x \geq d_{r} .
$$

If $d_{r} \notin D_{+}(\xi)$, that is $d_{r}=c_{r}$ and $d_{r} \in D_{-}(\xi)$, set

$$
f^{\#, \varepsilon}(x)= \begin{cases}f\left(d_{r}\right)=f(\hat{x}) & \text { for } x \in\left[d_{r}, d_{r}+\varepsilon\right], \\ f(x-\varepsilon) & \text { for } x \in\left[d_{r}+\varepsilon, b_{r}\right], \\ f(x)+f\left(b_{r}-\varepsilon\right)-f\left(b_{r}\right) & \text { for } x \in \Omega, x \geq b_{r} .\end{cases}
$$

We call the function $f^{\#, \varepsilon}$ an upper canonical modification of $f$ at $\hat{x}$ with an effective region $M$ and a canonical neighborhood $\Omega_{2}$. By a similar way we are able to construct a lower canonical modification $f_{\#, \varepsilon}$ with an effective region $M$ and a canonical neighborhood $\Omega_{2}$ : Let $-f_{\#, \varepsilon}$ be an upper canonical modification of $-f$ at $\hat{x}$.

The figures below illustrate how to construct the effective region $M$ and the upper canonical modification $f^{\#}=f^{\#, \varepsilon}$ when $f$ is $P$-faceted at $\hat{x}$ and $\chi_{l}=\chi_{r}=-1$. While Figure $\square$ indicates the case $d_{l} \in D_{-}(\xi)$ and $d_{r} \in D_{+}(\xi)$, Figure $\square$ shows the cases $d_{l} \in D_{-}(\xi)$ and $d_{r} \notin D_{+}(\xi)$.

The upper and lower canonical modification fulfills

Proposition 3.2. Assume $(W)$. Let $\Omega_{1}=\left(a_{l}, a_{r}\right) \subset \Omega$ be an open interval. For $f \in C(\Omega) \cap C_{P}^{2}\left(\Omega_{1}\right)$ and $\hat{x} \in \Omega_{1}$ satisfying $f^{\prime}(\hat{x})=0$, let $f^{\varepsilon}$ be an upper canonical modification $f^{\#, \varepsilon}$ (resp. lower canonical modification $f^{\#, \varepsilon}$ ) with effective region $M=\left[d_{l}, d_{r}\right]$ and a canonical neighborhood $\Omega_{2}=\left(b_{l}, b_{r}\right)$ and let $s=1$ (resp. $s=$ $-1)$. Let $y, y_{\varepsilon} \in M, y_{k} \in \Omega$ and equi-Lipschitz functions $\sigma, \sigma_{k}$ satisfy $y_{\varepsilon} \rightarrow y$, $y_{k} \rightarrow y$ and $\sigma_{k} \rightarrow \sigma$ uniformly. Then the conditions

$$
\begin{gathered}
f^{\varepsilon} \in C(\Omega) \cap C_{P}^{2}\left(\Omega_{2}\right), \\
s f^{\varepsilon}>s f \quad \text { on } \Omega \backslash M, \\
\inf _{\Omega \backslash \Omega_{2}} s\left(f^{\varepsilon}-f\right)>0, \\
f^{\varepsilon}(y)=f(y)=f(\hat{x}), \\
\lim _{k}\left(f^{\varepsilon}\right)^{\prime}\left(y_{k}\right)=\left(f^{\varepsilon}\right)^{\prime}(y), \\
\left(f^{\varepsilon}\right)^{\prime}(y)=f^{\prime}(y)=f^{\prime}(\hat{x})=0,
\end{gathered}
$$




$$
\limsup _{k} s \Lambda_{W}^{\sigma_{k}}\left(f^{\varepsilon}\right)\left(y_{k}\right) \leq s \Lambda_{W}^{\sigma}\left(f^{\varepsilon}\right)(y)
$$

hold for all $\varepsilon>0$ small enough, and

$$
\begin{gathered}
\Lambda_{W}^{\sigma}\left(f^{\varepsilon}\right)\left(y_{\varepsilon}\right) \rightarrow \Lambda_{W}^{\sigma}(f)(y) \quad \text { as } \varepsilon \rightarrow 0, \\
s \Lambda_{W}^{\sigma}(f)(y) \leq s \Lambda_{W}^{\sigma}(f)(\hat{x})
\end{gathered}
$$

hold.

Proof. We only consider the case $f^{\varepsilon}=f^{\#, \varepsilon}$ and $s=1$. Since it is easy to verify the conditions (B.3) $-\left(\right.$ (B.]) in the case (i) $f^{\prime}(\hat{x})=0 \notin P$, we only consider the case (ii) $f$ is $P$-faceted at $\hat{x}$. The conditions $(\mathbf{B . 3})-(\mathbf{B . 8})$ are shown by the definition of the canonical modification.

Show (B.9). Take a subsequence $k_{j}$ such that

$$
\Lambda_{W}^{\sigma_{k_{j}}}\left(f^{\#, \varepsilon}\right)\left(y_{k_{j}}\right) \rightarrow \underset{k}{\limsup } \Lambda_{W}^{\sigma_{k}}\left(f^{\#, \varepsilon}\right)\left(y_{k}\right) .
$$

Since Proposition $[2.7$ implies

$$
\Lambda_{W}^{\sigma_{k_{j}}}\left(f^{\#, \varepsilon}\right)\left(y_{k_{j}}\right) \rightarrow \Lambda_{W}^{\sigma}\left(f^{\#, \varepsilon}\right)(y)
$$

provided that $y_{k_{j}} \in R^{\varepsilon}=\left[c_{l}^{\varepsilon}, c_{r}^{\varepsilon}\right]:=R\left(f^{\#, \varepsilon}, \hat{x}\right)$ for each $j$, we may assume that $y_{k_{j}} \notin R^{\varepsilon}$. Also it is enough to consider the case $y_{k_{j}}<c_{l}^{\varepsilon}$. Hence,

$$
\begin{aligned}
\Lambda_{W}^{\sigma_{k_{j}}}\left(f^{\#, \varepsilon}\right)\left(y_{k_{j}}\right) & =W^{\prime \prime}\left(\left(f^{\#, \varepsilon}\right)^{\prime}\left(y_{k_{j}}\right)\right)\left(f^{\#, \varepsilon}\right)^{\prime \prime}\left(y_{k_{j}}\right)+\sigma_{k_{j}}\left(y_{k_{j}}\right) \\
& \rightarrow \sigma(y) .
\end{aligned}
$$

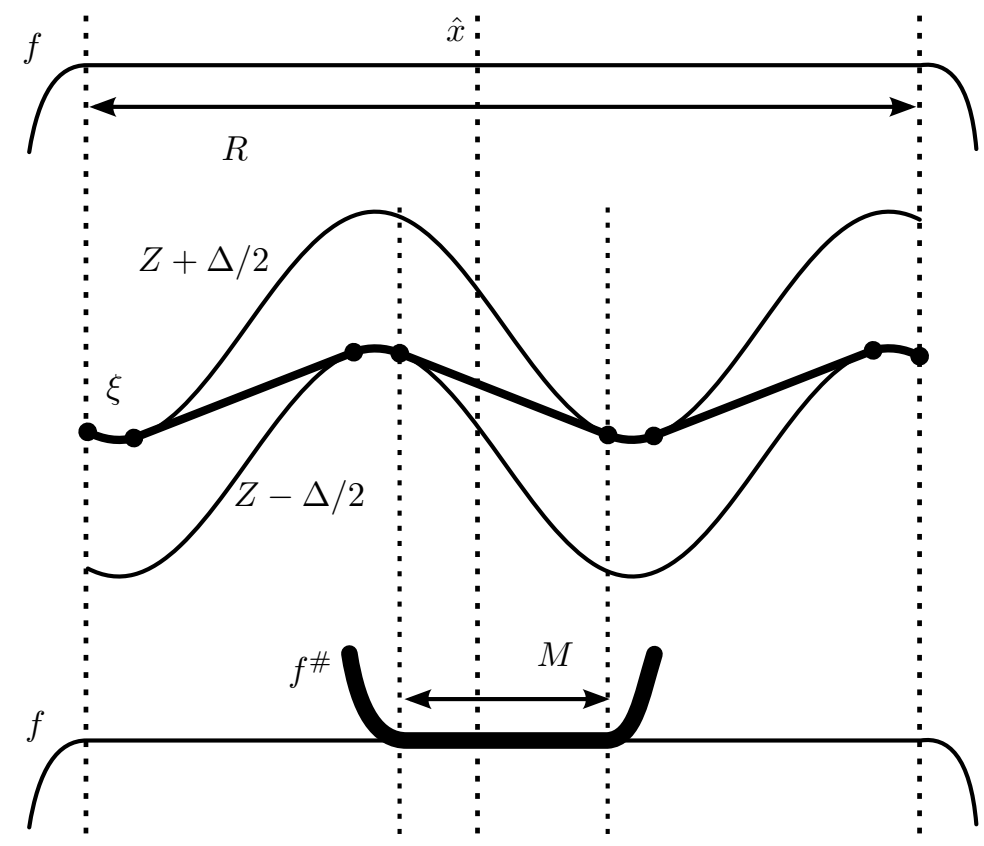

Figure 1. Construction of $M$ and $f^{\#}=f^{\#, \varepsilon}$ (case $d_{l} \in D_{-}(\xi)$ and $\left.d_{r} \in D_{+}(\xi)\right)$ 


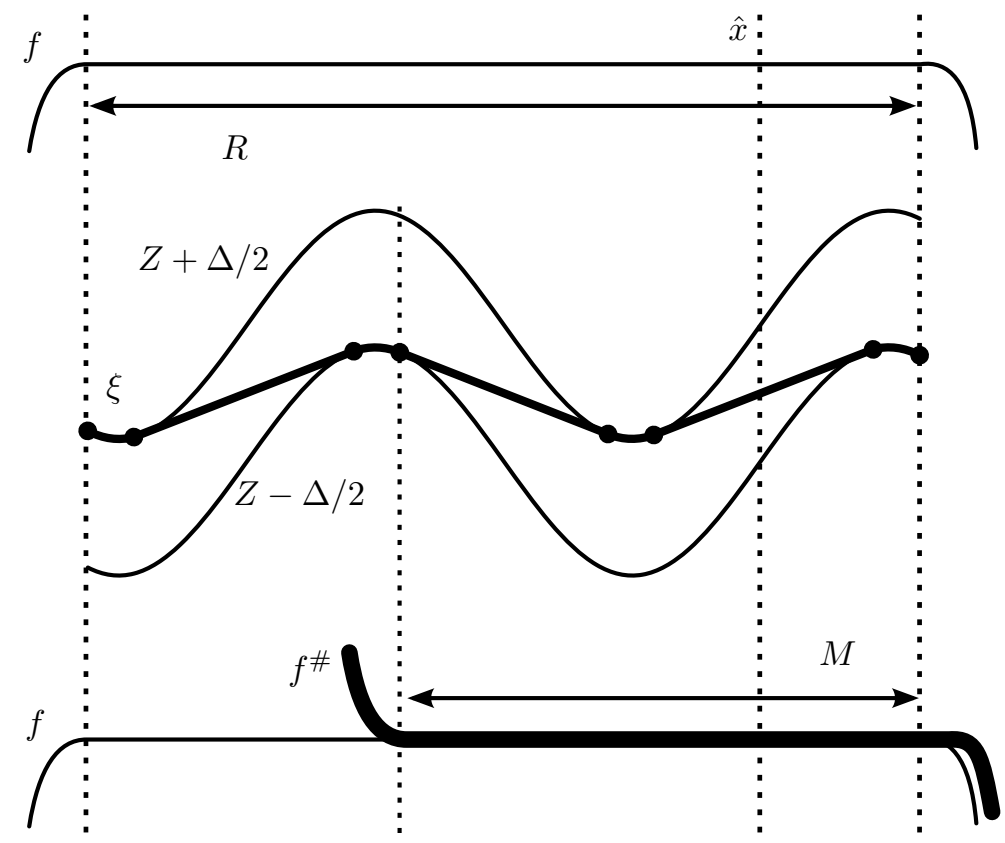

Figure 2. Construction of $M$ and $f^{\#}=f^{\#, \varepsilon}$ (case $d_{l} \in D_{-}(\xi)$ and $\left.d_{r} \notin D_{+}(\xi)\right)$

Since $y_{k_{j}} \rightarrow y \in M \subset R^{\varepsilon}$, we observe that

$$
y=c_{l}^{\varepsilon}=d_{l} \in D_{-}\left(\xi^{\varepsilon}\right),
$$

where $\xi^{\varepsilon}$ is the minimizer of the obstacle problem $\xi_{\chi_{l}^{\prime} \chi_{r}^{\prime}}^{Z, R^{\varepsilon}}$ with a primitive $Z$ of $\sigma$, $\Delta=|\partial W(0)|, R^{\varepsilon}=, \chi_{l}^{\prime}=\chi_{l}\left(f^{\#, \varepsilon}, \hat{x}\right), \chi_{r}^{\prime}=\chi_{r}\left(f^{\#, \varepsilon}, \hat{x}\right)$. Noting that $\xi^{\varepsilon}-Z+\Delta / 2$ attains zero minimum at $y$, we have

$$
\sigma(y) \leq \Lambda_{W}^{\sigma}\left(f^{\#, \varepsilon}\right)(y)
$$

and hence

$$
\limsup _{k} \Lambda_{W}^{\sigma_{k}}\left(f^{\varepsilon}\right)\left(y_{k}\right)=\lim _{j} \Lambda_{W}^{\sigma_{k_{j}}}\left(f^{\varepsilon}\right)\left(y_{k_{j}}\right) \leq \Lambda_{W}^{\sigma}\left(f^{\#, \varepsilon}\right)(y) .
$$

Show (B.]U) . Write $R=R(f, \hat{x}), \chi_{l}=\chi_{l}(f, \hat{x}), \chi_{r}=\chi_{r}(f, \hat{x})$ so that $\xi=\xi_{\chi_{l} \chi_{r}}^{Z, \Delta, R}$. Also note that $\chi_{l}^{\prime}$ and $\chi_{r}^{\prime}$ are independent of $\varepsilon$. It follows from Corollary 2.4 that

$$
\Lambda_{W}^{\sigma}(f)(y)=\Lambda_{\chi_{l} \chi_{r}}^{\sigma}(y ; R, \Delta)=\Lambda_{\chi_{l}^{\prime} \chi_{r}^{\prime}}^{\sigma}(y ; M, \Delta) .
$$

Since $R^{\varepsilon} \rightarrow M$ as $\varepsilon \rightarrow 0$, we see by Proposition 2.8 that

$$
\Lambda_{W}^{\sigma}\left(f^{\#, \varepsilon}\right)\left(y_{\varepsilon}\right)=\Lambda_{\chi_{l}^{\prime} \chi_{r}^{\prime}}^{\sigma}\left(y_{\varepsilon} ; R^{\varepsilon}, \Delta\right) \rightarrow \Lambda_{\chi_{l}^{\prime} \chi_{r}^{\prime}}^{\sigma}(y ; M, \Delta)=\Lambda_{W}^{\sigma}(f)(y) .
$$

Since $y \notin D_{-}(\xi)$ for all $y \in\left[\hat{x}, d_{r}\right)$, we see that $\xi$ is concave on $\left[\hat{x}, d_{r}\right]$ by the concave-convex condition of $\xi$. By a similar argument we see that $\xi$ is convex on $\left[d_{l}, \hat{x}\right]$. Therefore we obtain (B.]) for all $y \in M$. 


\section{Perron type existence theorem}

In this section we show Perron type existence theorem. Let $\Omega$ be an open set in $\mathbf{R}$ and $\mathcal{Q}=(0, T) \times \Omega$.

Theorem 4.1 (Perron type existence). Assume (W), (S), (F1), (F2). Let $u^{-}$and $u^{+}$respectively be a subsolution and a supersolution of (2]) satisfying

$$
u^{-} \leq u^{+} \text {in } \mathcal{Q}, \quad\left(u^{-}\right)_{*}>-\infty,\left(u^{+}\right)^{*}<\infty \text { on }[0, T) \times \Omega .
$$

(1) Then, there exists a solution $u$ of (区.]) satisfying

$$
u^{-} \leq u \leq u^{+} \quad \text { in } \mathcal{Q} \text {. }
$$

(2) Moreover, if

$$
\begin{gathered}
\sigma(t, x+\omega)=\sigma(t, x) \\
u^{-}(t, x+\omega)=u^{-}(t, x), \quad u^{+}(t, x+\omega)=u^{+}(t, x)
\end{gathered}
$$

for all $(t, x) \in \mathcal{Q}$ with $\omega>0$ and $\Omega=\mathbf{R}$, then there exists a solution $u$ of (‥]) satisfying (4.2) and

$$
u(t, x+\omega)=u(t, x) \quad \text { for all }(t, x) \in \mathcal{Q} .
$$

We divide the main part of the proof into two lemmas.

Lemma 4.2. Assume $(W),(S),(F 1),(F 2)$. Let $\mathcal{S}$ be a nonempty family of subsolutions (resp. supersolutions) of ([2.]). Define

$$
u(t, x)=\sup \{v(t, x) \mid v \in \mathcal{S}\} \quad(\operatorname{resp} . v(t, x)=\inf \{v(t, x) \mid v \in \mathcal{S}\})
$$

for $(t, x) \in \mathcal{Q}$. Assume that $u^{*}<\infty$ (resp. $\left.v_{*}>-\infty\right)$ in $[0, T) \times \bar{\Omega}$. Then $u$ is a subsolution (resp. supersolution) of (ए.]).

Lemma 4.3. Assume $(W),(S),(F 1),(F 2)$. Let $\mathcal{S}$ be the set of all subsolutions $u$

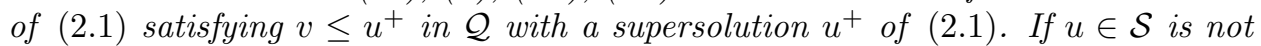
a supersolution of $(\mathbb{2 . ]})$ and satisfies $u_{*}>-\infty$ in $[0, \infty) \times \Omega$, then there exist a function $v \in \mathcal{S}$ and a point $(s, y) \in \mathcal{Q}$ such that $u(s, y)<v(s, y)$.

We first show the Perron type existence theorems under the assumption that Lemma 4.2 and 4.3 hold.

Proof of Theorem 4.1 . we shall show the part (1). Let $\mathcal{S}$ be the set of all subsolutions $v$ of $([\mathbb{E}])$ satisfying $v \leq u^{+}$in $\mathcal{Q}$. Note that $\mathcal{S}$ is not empty since $u^{-} \in \mathcal{S}$. Define

$$
u(t, x)=\sup \{v(t, x) \mid v \in \mathcal{S}\} \quad \text { for }(t, x) \in \mathcal{Q} .
$$

We then have $u^{-} \leq u \leq u^{+}$in $\mathcal{Q}$, which implies $u_{*} \geq\left(u^{-}\right)_{*}>-\infty$ and $u^{*} \leq$ $\left(u^{+}\right)^{*}<\infty$ on $[0, T) \times \Omega$. We hence see that $u$ is a subsolution by Proposition 4.2 . We next claim that $u$ is a supersolution. If $u$ were not a supersolution, Proposition 4.3 would imply that there exist $v \in \mathcal{S}$ and $(s, y) \in \mathcal{Q}$ such that $u(s, y)<v(s, y)$, which contradicts the maximality of $u$. Therefore, we conclude that $u$ is a solution.

It remains to show (4.5). Note that for $v \in \mathcal{S}$ the periodicity conditions (4.3) and (4.4) imply that $\tilde{v}(t, x)=v(t, x \pm \omega) \in \mathcal{S}$. Hence, we see that

$$
u(t, x+\omega)=\sup \{v(t, x+\omega) \mid v \in \mathcal{S}\}=\sup \{v(t, x) \mid v \in \mathcal{S}\}=u(t, x) .
$$

The proof is now complete. 
We next show the lemmas. We note that being a subsolution is equivalent to being a subsolution in the local sense by Lemma 2.14 .

Proof of Lemma L.g. We only show that $u$ is a subsolution (in the local sense). Fix a point $(\hat{t}, \hat{x}) \in \mathcal{Q}$ and a locally admissible test function $\varphi \in C(\mathcal{Q})$ at $(\hat{t}, \hat{x})$ such that (2.6) holds. Our goal is to show (ए.D). Since $\varphi$ is locally admissible, there exist $f \in C_{P}^{2}\left(\Omega_{1}\right)$ and $g \in C^{1}(I)$ with open intervals $\Omega_{1}$ and $J$ such that

$$
\begin{aligned}
& \varphi(t, x)=f(x)+g(t) \quad \text { on } \mathcal{Q}_{1}:=J \times \Omega_{1}, \\
& R(f, \hat{x}) \subset \Omega_{1} \subset \Omega, \quad \hat{t} \in J \subset(0, T) .
\end{aligned}
$$

We may assume that

$$
\left(u^{*}-\varphi\right)(\hat{t}, \hat{x})=0, \quad \varphi_{x}(\hat{t}, \hat{x})=0
$$

Therefore, the desired inequality ( $[\mathrm{E}$ ) becomes

$$
g^{\prime}(\hat{t})+F\left(\hat{t}, 0, \Lambda_{W}^{\sigma(\hat{t})}(f)(\hat{x})\right) \leq 0,
$$

which we should show.

We now let $\psi \in C(\mathcal{Q})$ be an $A_{P}\left(\mathcal{Q}_{2}\right)$ function such that

$$
\psi=\varphi \text { on } K, \quad \psi>\varphi \text { on } \mathcal{Q} \backslash K, \quad \inf _{\mathcal{Q} \backslash \mathcal{Q}_{2}}(\psi-\varphi)>0
$$

with a closed set $K$ and an open set $\mathcal{Q}_{2}$ satisfying

$$
(\hat{x}, \hat{t}) \in K \subset \mathcal{Q}_{2} \subset \mathcal{Q} .
$$

The function $\psi$ is to be determined later. By the definition of the upper semicontinuous envelope there exists a sequence $\left\{\left(t_{k}, s_{k}\right)\right\}_{k \in \mathbf{N}} \subset \mathcal{Q}_{2}$ such that

$$
\left(t_{k}, x_{k}, u\left(t_{k}, x_{k}\right)\right) \rightarrow\left(\hat{t}, \hat{x}, u^{*}(\hat{t}, \hat{x})\right) \quad \text { as } k \rightarrow \infty .
$$

By the definition of $u$ there exists $\left\{v_{k}\right\}_{k \in \mathbf{N}} \subset \mathcal{S}$ such that

$$
v_{k}\left(t_{k}, x_{k}\right)>u\left(t_{k}, x_{k}\right)-1 / k
$$

and so

$$
v_{k}\left(t_{k}, x_{k}\right) \rightarrow u^{*}(\hat{t}, \hat{x}) \quad \text { as } k \rightarrow \infty .
$$

Taking a maximizer $\left(s_{k}, y_{k}\right)$ of $v_{k}^{*}-\psi$ on $\overline{\mathcal{Q}_{2}}$, we observe that

$$
\left(\left(v_{k}\right)^{*}-\psi\right)\left(t_{k}, x_{k}\right) \leq\left(\left(v_{k}\right)^{*}-\psi\right)\left(s_{k}, y_{k}\right) \leq\left(u^{*}-\psi\right)\left(s_{k}, y_{k}\right)
$$

for each $k$. Sending $k \rightarrow \infty$ yields

$$
\left(u^{*}-\psi\right)(\hat{t}, \hat{x}) \leq\left(u^{*}-\psi\right)(\bar{s}, \bar{y})
$$

where

$$
(\bar{s}, \bar{y})=\lim _{k \rightarrow \infty}\left(s_{k}, y_{k}\right) \in \overline{\mathcal{Q}_{2}}
$$

by taking a subsequence if necessary. We see that $(\bar{s}, \bar{y}) \in K$ and $\left(s_{k}, y_{k}\right) \in \mathcal{Q}_{2}$ for sufficiently large $k$. We also note that

$$
\max _{\mathcal{Q}}\left(\left(v_{k}\right)^{*}-\psi\right)=\left(\left(v_{k}\right)^{*}-\psi\right)\left(s_{k}, y_{k}\right),
$$

i.e. $\psi$ is a test function of $v_{k}$ at $\left(s_{k}, y_{k}\right)$ by the last inequality of (4.8).

Let $f^{\#, \varepsilon}$ be an upper canonical modification of $f$ at $\hat{x}$ with effective region $M$ and canonical neighborhood $\Omega_{2} \subset \Omega_{1}$ for $\varepsilon>0$. We then see that

$$
\psi(x, t)=f^{\#, \varepsilon}(x)+g(t)+(t-\hat{t})^{2}
$$


is an admissible function on a set $\mathcal{Q}_{2}=J \times \Omega_{2} \subset \mathcal{Q}_{1}$ and that (4.8) holds with $K=\{\hat{t}\} \times M$ by Proposition $\left[2\right.$. By the above argument we have $v_{k}^{\varepsilon} \in \mathcal{S}$ and $\left(s_{k}^{\varepsilon}, y_{k}^{\varepsilon}\right) \in \mathcal{Q}_{2}$ such that

$$
\left(s_{k}^{\varepsilon}, y_{k}^{\varepsilon}\right) \rightarrow\left(\hat{t}, y^{\varepsilon}\right) \in\{\hat{t}\} \times M \quad \text { as } k \rightarrow \infty
$$

and $\psi$ is a test function of $v_{k}^{\varepsilon}$ at $\left(s_{k}^{\varepsilon}, y_{k}^{\varepsilon}\right)$. Since $v_{k}^{\varepsilon}$ is a subsolution, we have

$$
g^{\prime}\left(s_{k}^{\varepsilon}\right)+2\left(s_{k}^{\varepsilon}-\hat{t}\right)+F\left(s_{k}^{\varepsilon},\left(f^{\#, \varepsilon}\right)^{\prime}\left(y_{k}^{\varepsilon}\right), \Lambda_{W}^{\sigma\left(s_{k}^{\varepsilon}\right)}\left(f^{\#, \varepsilon}\right)\left(y_{k}^{\varepsilon}\right)\right) \leq 0 .
$$

Proposition 3.2 implies that

$$
\begin{aligned}
\lim _{k \rightarrow \infty}\left(f^{\#, \varepsilon}\right)^{\prime}\left(y_{k}^{\varepsilon}\right) & =f^{\prime}(\hat{x}), \\
\lim _{\varepsilon \rightarrow 0} \limsup _{k \rightarrow \infty} \Lambda_{W}^{\sigma\left(s_{k}^{\varepsilon}\right)}\left(f^{\#, \varepsilon}\right)\left(y_{k}^{\varepsilon}\right) & \leq \Lambda_{W}^{\sigma(\hat{t})}(f)(\hat{x}) .
\end{aligned}
$$

Therefore, it follows from (4.9) that (4.7) holds by (F1) and (F2).

We conclude that $u$ is a subsolution.

Proof of Lemma 4. S. Since $u$ is not a supersolution, there exist $(\hat{x}, \hat{t}) \in \mathcal{Q}$ and a locally admissible test function $\varphi \in C(\mathcal{Q})$ at $(\hat{t}, \hat{x})$ such that $(\mathbb{E . G})$ and

$$
\varphi_{t}(\hat{t}, \hat{x})+F\left(\hat{t}, \varphi_{x}(\hat{t}, \hat{x}), \Lambda_{W}^{\sigma(\hat{t})}(\varphi(\hat{t}, \cdot))(\hat{x})\right)<0
$$

hold. Since $\varphi$ is locally admissible, there exist $f \in C_{P}^{2}\left(\Omega_{1}\right)$ and $g \in C^{1}(J)$ with open intervals $\Omega_{1}$ and $J$ such that (4.6) holds with $\mathcal{Q}_{1}:=J \times \Omega_{1}$. We may assume that

$$
\left(u_{*}-\varphi\right)(\hat{t}, \hat{x})=0, \quad \varphi_{x}(\hat{t}, \hat{x})=0
$$

by Proposition [.]2 with $a=\varphi_{x}(\hat{t}, \hat{x})=f^{\prime}(\hat{x})$ and $b=u_{*}(\hat{x}, \hat{t})-f^{\prime}(\hat{x}) \hat{x}$. Therefore, the inequality (4.]1) becomes

$$
g^{\prime}(\hat{t})+F\left(\hat{t}, 0, \Lambda_{W}^{\sigma(\hat{t})}(f)(\hat{x})\right)<0 .
$$

Take a lower canonical modification $f_{\#, \varepsilon}$ of $f$ at $\hat{x}$ for $\varepsilon>0$ with effective region $M$ and canonical neighborhood $\Omega_{2} \subset \Omega_{1}$. Set

$$
\psi(x, t)=f_{\#, \varepsilon}(x)+g(t)-(t-\hat{t})^{2} .
$$

We now claim that

$$
\psi_{t}(t, x)+F\left(t, \psi_{x}(t, x), \Lambda_{W}^{\sigma(t)}(\varphi(t, \cdot))(x)\right)<0,
$$

i.e.

$$
g^{\prime}(t)-2(t-\hat{t})+F\left(t,\left(f_{\#, \varepsilon}\right)^{\prime}(x), \Lambda_{W}^{\sigma(t)}\left(f_{\#, \varepsilon}\right)(x)\right)<0
$$

for all $(t, x)$ in some neighborhood of $K:=\{\hat{t}\} \times M$ choosing $\varepsilon$ small enough. Indeed, since Proposition 5.2 implies that

$$
\lambda^{\varepsilon}(t, x):=\Lambda_{W}^{\sigma(t)}\left(f_{\#, \varepsilon}\right)(x)
$$

is lower semicontinuous at each point of the compact set $K$, we see that for every $m>0$ there exists an open set $\mathcal{Q}_{3} \supset K$ on which the inequality

$$
\lambda^{\varepsilon}(t, x)>\min _{K} \lambda^{\varepsilon}-m
$$

holds. Choose $y_{\varepsilon} \in M$ such that $\left(\hat{t}, y_{\varepsilon}\right)$ is a minimum point of $\lambda^{\varepsilon}$ on $K=\{\hat{t}\} \times M$. Proposition 5.2 implies that

$$
\Lambda_{W}^{\sigma(t)}\left(f_{\#, \varepsilon}\right)(x)>\Lambda_{W}^{\sigma(\hat{t})}(f)(\hat{x})-m
$$


for all $(t, x) \in \mathcal{Q}_{3}$ with small $\varepsilon$ and $m$. Since Proposition 3.2 also implies that

$$
\left|\left(f_{\#, \varepsilon}\right)^{\prime}(x)\right|<m,
$$

it follows from (4.9) that (4..3) and so (4..2) holds on $\mathcal{Q}_{3}$ by $(\mathrm{F} 1)$ and (F2).

We next claim that $\psi<\left(u^{+}\right)_{*}$ in $\mathcal{Q}_{3}$. First note that $\psi \leq \varphi \leq u \leq u^{+}$and so $\psi \leq\left(u^{+}\right)_{*}$. If $\psi(t, x)=\left(u^{+}\right)_{*}(t, x)$ at some point $(t, x) \in \mathcal{Q}_{3}$, then $\psi$ would be a test function of the supersolution $u^{+}$at $(t, x)$. Hence,

$$
\psi_{t}(t, x)+F\left(t, \psi_{x}(t, x), \Lambda_{W}^{\sigma(t)}(\psi(t, \cdot))(x)\right) \geq 0
$$

which contradicts to (4.T2).

Take a bounded open set $\mathcal{Q}_{4}$ such that $K \subset \mathcal{Q}_{4}$ and $\overline{\mathcal{Q}_{4}} \subset \mathcal{Q}_{3}$. Letting $\sigma_{1}=$ $\inf _{\mathcal{Q}_{4}}\left(\left(u^{+}\right)_{*}-\psi\right)>0$, we have

$$
\psi+\sigma_{1} \leq\left(u^{+}\right)_{*} \text { in } \mathcal{Q}_{4} .
$$

Since $f_{\#, \varepsilon}<f$ on $\Omega_{2} \backslash M$ by Proposition 3.2, we also have

$$
\psi+\sigma_{2} \leq u_{*} \quad \text { in } \mathcal{Q}_{3} \backslash \mathcal{Q}_{4}
$$

with $\sigma_{2}=\inf _{\mathcal{Q}_{3} \backslash \mathcal{Q}_{4}}\left(u_{*}-\psi\right)>0$. Define a function $v$ by

$$
v(t, x)= \begin{cases}\max \{\psi(t, x)+\sigma, u(t, x)\} & \text { for }(t, x) \in \mathcal{Q}_{3}, \\ u(t, x) & \text { for }(t, x) \notin \mathcal{Q}_{3} .\end{cases}
$$

with $\sigma=\min \left\{\sigma_{1}, \sigma_{2}\right\}$. We show that this function $v$ is a desirable function in the statement of this lemma.

Note that $v \geq u$. In addition, since $\left(u_{*}-\psi\right)(\hat{t}, \hat{x})=0$, there exists $(s, y) \in \mathcal{Q}_{4}$ such that $(u-\psi)(s, y)<\sigma$, which implies

$$
v(s, y)>u(s, y) .
$$

Since

$$
\psi(t, x)+\sigma \leq \begin{cases}\left(u^{+}\right)_{*}(t, x) & \text { if }(t, x) \in \mathcal{Q}_{4}, \\ u_{*}(t, x) & \text { if }(t, x) \in \mathcal{Q}_{3} \backslash \mathcal{Q}_{4},\end{cases}
$$

and $u \leq u^{+}$in $\mathcal{Q}$, we have

$$
\begin{gathered}
v=u \quad \text { on } \mathcal{Q} \backslash \mathcal{Q}_{4}, \\
v \leq u^{+} \quad \text { in } \mathcal{Q} .
\end{gathered}
$$

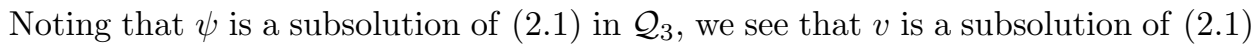
in $\mathcal{Q}_{3}$ by Lemma [2.] and 4.2. Therefore, if we admit the next lemma, we have $w \in \mathcal{S}$ and so the proof is finished.

Lemma 4.4. Assume (W), (S), (F1), (F2). Let u be a subsolution of (एत) in $\mathcal{Q}$. Let $w$ be a function defined on $\mathcal{Q}$ such that $w \geq u$ in $\mathcal{Q}, w=u$ in $\mathcal{Q} \backslash \overline{N_{2}}$, and $w$ is a subsolution of (ए.]) in $N_{1}$ with open rectangle sets $N_{1}=J_{1} \times I_{1}, N_{2}=J_{2} \times I_{2}$

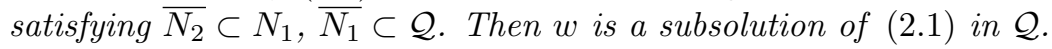

In the classical setting, say $P=\emptyset$, this is easy to prove; if a function is a solution in two domains, then it is a solution in their union. However, this assertion on locality of solutions is not true for our equation (एत) in general. 
Proof. Fix a point $(\hat{t}, \hat{x}) \in \mathcal{Q}$ and a locally admissible test function $\varphi \in C(\mathcal{Q})$ of $w$ at $(\hat{t}, \hat{x})$, i.e. $\max _{\mathcal{Q}}\left(w^{*}-\varphi\right)=\left(w^{*}-\varphi\right)(\hat{t}, \hat{x})$. Since $\varphi$ is locally admissible, there exist $f \in C_{P}^{2}\left(\Omega_{1}\right)$ and $g \in C^{1}(J)$ with open intervals $\Omega_{1}$ and $J$ such that (4.6) holds. We may assume that

$$
\left(w^{*}-\varphi\right)(\hat{t}, \hat{x})=0, \quad \varphi_{x}(\hat{t}, \hat{x})=0
$$

by Proposition 2.2 with $a=\varphi_{x}(\hat{t}, \hat{x})=f^{\prime}(\hat{x})$ and $b=w^{*}(\hat{x}, \hat{t})-f^{\prime}(\hat{x}) \hat{x}$. We should show (4.7).

It is enough to consider the case

$$
(\hat{t}, \hat{x}) \in N_{2}, \quad \varphi(\hat{t}, \hat{x})>u(\hat{t}, \hat{x}) ;
$$

otherwise, $\varphi$ is a test of the subsolution $u$ and so we have (4.7). We may also assume that $f$ is $P$-faceted at $\hat{x}$ and $R(f, \hat{x})$ is not contained by $I_{1}$; otherwise, (4.Z) holds since $w$ is a subsolution in $N_{1}$.

Let $f^{\#}=f^{\#, \varepsilon}$ be a upper canonical modification of $f$ at $\hat{x}$ with effective region $M$ and canonical neighborhood $\Omega_{2} \subset \Omega_{1}$. Set

$$
\psi(x, t)=f^{\#}(x)+g(t)+(t-\hat{t})^{2} .
$$

We then observe that

$$
\psi>\varphi \geq w^{*} \geq u^{*} \quad \text { in } \mathcal{Q} \backslash\{\hat{t}\} \times M .
$$

Let us assume for the moment that $\psi\left(\hat{t}, x_{0}\right)=u^{*}\left(\hat{t}, x_{0}\right)$ at some $x_{0} \in M$. Then, since $\psi$ is a test function of the subsolution $u$ at $\left(\hat{t}, x_{0}\right)$, we have

$$
g^{\prime}(\hat{t})+F\left(\hat{t},\left(f^{\#}\right)^{\prime}\left(x_{0}\right), \Lambda_{W}^{\sigma(\hat{t})}\left(f^{\#}\right)\left(x_{0}\right)\right) \leq 0 .
$$

Proposition $\$ .2$ yields (4.7) by (F2). Therefore, we have

$$
\psi>u^{*} \text { in } \mathcal{Q}
$$

We now take a faceted function whose faceted region is contained in $I_{1}$; set

$$
\tilde{f}^{\#}(x)= \begin{cases}f^{\#}(x)+k\left|x-c_{l}\right|^{3}\left(\left|x-c_{l}\right|-1\right) & \text { for } x \in \Omega, x \leq c_{l} \\ f^{\#}(x) & \text { for } x \in\left[c_{l}, c_{r}\right] \\ f^{\#}(x)+k\left|x-c_{r}\right|^{3}\left(\left|x-c_{r}\right|-1\right) & \text { for } x \in \Omega, x \geq c_{r},\end{cases}
$$

where $I_{2} \subset\left[c_{l}, c_{r}\right] \subset I_{1}$ and $k>0$. Note that

$$
\tilde{\psi}(t, x):=\tilde{f}^{\#}(x)+g(t)+(t-\hat{t})^{2}
$$

is locally admissible in $N_{1}$. Taking $k$ small enough, we have

$$
\tilde{\psi}>u^{*} \text { for }(t, x) \in \mathcal{Q}
$$

by (4.14). Noting that

$$
w=u \text { in } \mathcal{Q} \backslash \overline{N_{2}}, \quad \tilde{\psi}=\psi \text { in } I_{1} \times\left[c_{l}, c_{r}\right] \supset \overline{N_{2}},
$$

we see that $\max _{\mathcal{Q}}\left(w^{*}-\tilde{\psi}\right)=\left(w^{*}-\tilde{\psi}\right)(\hat{t}, \hat{x})$. Since $\tilde{\psi}$ is a test function,

$$
g^{\prime}(\hat{t})+F\left(\hat{t},\left(\tilde{f}^{\#}\right)^{\prime}(\hat{x}), \Lambda_{W}^{\sigma(\hat{t})}\left(\tilde{f}^{\#}\right)(\hat{x})\right) \leq 0 .
$$

Note that Proposition [2.6 yields

$$
\Lambda_{W}^{\sigma(\hat{t})}\left(\tilde{f}^{\#}\right)(\hat{x}) \leq \Lambda_{W}^{\sigma(\hat{t})}\left(f^{\#}\right)(\hat{x}) .
$$

Therefore, we have

$$
g^{\prime}(\hat{t})+F\left(\hat{t},\left(f^{\#}\right)^{\prime}(\hat{x}), \Lambda_{W}^{\sigma(\hat{t})}\left(f^{\#}\right)(\hat{x})\right) \leq 0,
$$


ON EXISTENCE RESULTS FOR ONE-DIMENSIONAL SINGULAR DIFFUSION EQUATIONSI7

which gives (4.7).

\section{EXISTENCE THEOREM FOR PERIODIC INITIAL DATA}

In this section we prove an existence theorem for the equation (ए.]) with periodic boundary condition and initial condition. In order to utilize the Perron type existence theorem (Theorem 4 . له ) we construct a subsolution $u^{-}$and a supersolution $u^{+}$with given initial data; for a general strategy; see [ㅍ].

Lemma 5.1 (Existence of sub- and supersolutions). Assume (W), (S), (F1), (F2) with $\Omega=\mathbf{R}$. Also assume that $u_{0}$ is a bounded and uniformly continuous function on $\mathbf{R}$ and $\sigma$ is bounded. Then, there exist an upper semicontinuous function $u^{+}$ and an lower semicontinuous function $u^{-}$on $\overline{\mathcal{Q}}$ such that $u^{+}$and $u^{-}$respectively are a supersolution and a subsolution of (区- $\mathbf{D})$ in $\mathcal{Q}$ and

$$
u^{-}(0, x)=u_{0}(x)=u^{+}(0, x), \quad u^{-}(t, x) \leq u_{0}(x) \leq u^{+}(t, x)
$$

holds for all $(t, x) \in \mathcal{Q}$. Moreover, if

$$
u_{0}(x+\omega)=u_{0}(x)
$$

then $u^{ \pm}$can be taken so that it is spatially periodic with period $\omega$, i.e. (4.4) holds.

We show this existence theorem as in [112, Section 9].

Lemma 5.2 ([12, Lemma 9.5]). For each $\delta \in(0,1 / 2)$ and $M>0$ there exists $V=V_{\delta, M} \in C_{P}^{2}(\mathbf{R})$ such that

$$
\begin{gathered}
V \geq 0, \quad V^{\prime \prime} \geq 0 \text { in } \mathbf{R}, \quad V(0)=0, \quad V(x) \geq M \text { for }|x|>\delta, \\
V^{\prime}(x)= \begin{cases}q & \text { for } x \leq-1, \\
q^{\prime} & \text { for } x \geq 1\end{cases}
\end{gathered}
$$

with some $q, q^{\prime} \notin P$.

We need to show

Lemma 5.3. Let $V \in C_{P}^{2}(\mathbf{R})$ be such that $V^{\prime \prime} \geq 0$ and (5.3) holds with some $q, q^{\prime} \notin P$. Then for $B \in \mathbf{R}$ large enough

$$
V^{+}(t, x)=B t+V(x)
$$

is a supersolution of (एत) in $(0, T) \times \mathbf{R}$.

Proof. We first claim that

$$
C:=\sup \left\{\left|\Lambda_{W}^{\sigma(t)}(V)(x)\right| \mid(t, x) \in \mathcal{Q}\right\}<\infty .
$$

Note that $V^{\prime}(x) \in\left[q, q^{\prime}\right]$ for $x \in \mathbf{R}$ and

$$
\sup _{\mathbf{R}}\left|V^{\prime \prime}\right|=\sup _{[-1,1]}\left|V^{\prime \prime}\right|<\infty .
$$

Moreover, we have

$$
\sup _{p \in\left[q, q^{\prime}\right] \backslash P}\left|W^{\prime \prime}(p)\right|<\infty, \quad \sup _{\mathcal{Q}}|\sigma|<\infty .
$$


Therefore, for each $(t, x) \in \mathcal{Q}$ with $V^{\prime}(x) \notin P$ we observe that

$$
\begin{aligned}
\left|\Lambda_{W}^{\sigma(t)}(V)(x)\right| & \leq\left|W^{\prime \prime}\left(V^{\prime}(x)\right)\right|\left|V^{\prime \prime}(x)\right|+|\sigma(t, x)| \\
& \leq \sup _{p \in\left[q, q^{\prime}\right] \backslash P}\left|W^{\prime \prime}(p)\right| \sup _{\mathbf{R}}\left|V^{\prime \prime}\right|+\sup _{\mathcal{Q}}|\sigma|<\infty .
\end{aligned}
$$

We shall show that

$$
c_{p}:=\sup \left\{\left|\Lambda_{W}^{\sigma(t)}(V)(x)\right| \mid(t, x) \in \mathcal{Q}, V^{\prime}(x)=p\right\}<\infty
$$

for each $p \in P$. Indeed, since a faceted region $R=\left\{x \in \mathbf{R} \mid V^{\prime}(x)=p\right\}$ is a bounded closed interval, Proposition $[$.$] implies that (t, x) \mapsto \Lambda_{W}^{\sigma(t)}(V)(x)$ is continuous on $[0, T] \times R$, and so $c_{p}<\infty$. We note that the number of faceted regions of $V$ is finite, i.e. $P \cap\left[q, q^{\prime}\right]$ is finite by $(\mathrm{W})$. Hence we have

$$
\sup \left\{\left|\Lambda_{W}^{\sigma(t)}(V)(x)\right| \mid(t, x) \in \mathcal{Q}, V^{\prime}(x) \in P\right\}=\sup _{p \in P \cap\left[q, q^{\prime}\right]} c_{p}<\infty .
$$

Combining (5. 5 ) and (5.7), we obtain (5.5). Moreover, we see that

$$
F\left(t, V^{\prime}(x), \Lambda_{W}^{\sigma(t)}(V)(x)\right) \geq \inf _{[0, T] \times\left[q, q^{\prime}\right] \times[-C, C]} F=:-B_{0}>-\infty .
$$

Therefore, $V^{+}$in (5.4) is a supersolution of (2.]) for $B \geq B_{0}$.

Proof of Lemma [.]. Let $\delta$ be a modulus of continuity of $u_{0} ; \delta$ is a continuous nondecreasing function on $[0, \infty)$ with $\delta(0)=0$ such that

$$
\left|u_{0}(x)-u_{0}(y)\right| \leq \delta(|x-y|) \quad \text { for } x, y \in \mathbf{R} .
$$

By Lemma 52 and 5.3 take $V_{\delta}=V_{\delta, M} \in C_{P}^{2}(\mathbf{R})$ and $B_{\delta} \geq 0$ for small $\delta$ and $M=$ $\max u_{0}-\min u_{0}$ satisfying (52) and that $V_{\delta}^{+}(t, x)=B_{\delta} t+V_{\delta}(x)$ is a supersolution of (‥1). Define

$$
u_{\varepsilon, \xi}^{+}(t, x):=V_{\delta(\varepsilon)}^{+}(t, x-\xi)+u_{0}(\xi)+\varepsilon
$$

for small $\varepsilon>0$ and $\xi \in \mathbf{R}$. Note that $u_{\varepsilon, \xi}^{+}$is a supersolution of (区्]) and

$$
u_{\varepsilon, \xi}^{+}(t, x) \geq V_{\delta(\varepsilon)}(x-\xi)+u_{0}(\xi)+\varepsilon .
$$

On the case $|\xi-x| \leq \delta(\varepsilon)$ we observe that

$$
u_{\varepsilon, \xi}^{+}(t, x) \geq u_{0}(\xi)+\varepsilon \geq u_{0}(x)
$$

on the other case

$$
u_{\varepsilon, \xi}^{+}(t, x) \geq M+u_{0}(\xi) \geq u_{0}(x) .
$$

Therefore, Lemma 4.2 implies that

$$
u^{+}(t, x):=\inf _{\varepsilon>0, \xi \in \mathbf{R}} u_{\varepsilon, \xi}^{+}(t, x)
$$

is an upper semicontinuous supersolution of (ए.]) satisfying $u^{+} \geq u_{0}$. Moreover, since

$$
u_{\varepsilon, x}^{+}(0, x)=u_{0}(x)+\varepsilon \rightarrow u_{0}(x) \quad \text { as } \varepsilon \rightarrow 0,
$$

we have $u^{+}(0, x)=u_{0}(x)$ for all $x \in \mathbf{R}$. Under the assumption that $u_{0}$ is periodic we see that

$$
\begin{aligned}
u^{+}(t, x+\omega) & =\inf _{\varepsilon>0, \xi \in \mathbf{R}}\left(V_{\delta(\varepsilon)}^{+}(t, x+\omega-\xi)+u_{0}(\xi)+\varepsilon\right) \\
& =\inf _{\varepsilon>0, \xi \in \mathbf{R}}\left(V_{\delta(\varepsilon)}^{+}(t, x-\xi)+u_{0}(\xi+\omega)+\varepsilon\right)=u^{+}(t, x) .
\end{aligned}
$$

The same proof is valid for existence of a subsolution $u^{-}$. 
ON EXISTENCE RESULTS FOR ONE-DIMENSIONAL SINGULAR DIFFUSION EQUATIONSI9

\section{Combining Theorem 4.1 and 5.1] we have}

Theorem 5.4 (Existence theorem for periodic initial data). Assume (W), (S), (F1), (F2) and (4.3) with $\Omega=\mathbf{R}$ and $\omega>0$. Let $u_{0}$ be a continuous function satisfying (5.]). Then there exists a solution $u$ of (․ㅣ) satisfying (4.5) and

$$
u(0, x)=u(x) \quad \text { for all } x \in \mathbf{R} .
$$

\section{ACKNOWLEDGEMENT}

The work of the second author has been partly supported by Japan Society for the Promotion of Science (JSPS) through grants for scientific research Kiban (S) 21224001, Kiban (A) 23244015 and 25610025. The work of the third author was supported by Leading Graduate School Doctoral Program from The Ministry of Education, Culture, Sports, Science and Technology, and by a Grant-in-Aid for JSPS Fellows No. 25-7077.

\section{REFERENCES}

[1] S. Angenent and M. E. Gurtin, Multiphase thermomechanics with interfacial structure. II. Evolution of an isothermal interface, Arch. Rational Mech. Anal. 108 (1989), no. 4, 323-391.

[2] J. W. Barrett, H. Garcke, and R. Nürnberg, Numerical computations of faceted pattern formation in snow crystal growth, Phys. Rev. E 86 (2012), no. 1, 011604.

[3] G. Bellettini, V. Caselles, A. Chambolle, and M. Novaga, Crystalline mean curvature flow of convex sets, Arch. Ration. Mech. Anal. 179 (2006), no. 1, 109-152.

[4] A. Chambolle and M. Novaga, Existence and uniqueness for planar anisotropic and crystalline curvature flow, Proc. of 'Variational Methods for Evolving Objects', (eds. Y. Giga, Y. Tonegawa and P. Rybka), Adv. Stud. Pure Math., to appear.

[5] A. Chambolle, An algorithm for mean curvature motion, Interfaces Free Bound. 6 (2004), no. 2, 195-218.

[6] Y. G. Chen, Y. Giga, and S. Goto, Uniqueness and existence of viscosity solutions of generalized mean curvature flow equations, J. Differential Geom. 33 (1991), no. 3, 749-786.

[7] M. G. Crandall, H. Ishii, and P.-L. Lions, User's guide to viscosity solutions of second order partial differential equations, Bull. Amer. Math. Soc. 27 (1992), no. 1, 1-67.

[8] C. M. Elliott, A. R. Gardiner, and R. Schätzle, Crystalline curvature flow of a graph in a variational setting, Adv. Math. Sci. Appl. 8 (1998), no. 1, 425-460.

[9] L. C. Evans and J. Spruck, Motion of level sets by mean curvature. I, J. Differential Geom. 33 (1991), no. 3, 635-681.

[10] T. Fukui and Y. Giga, Motion of a graph by nonsmooth weighted curvature, World Congress of Nonlinear Analysts '92, de Gruyter, Berlin, 1996, 47-56.

[11] M.-H. Giga and Y. Giga, A subdifferential interpretation of crystalline motion under nonuniform driving force, Discrete Contin. Dynam. Systems (1998), no. Added Volume I, 276-287.

[12] _ Evolving graphs by singular weighted curvature, Arch. Rational Mech. Anal. 141 (1998), no. 2, 117-198.

[13] Stability for evolving graphs by nonlocal weighted curvature, Comm. Partial Differential Equations 24 (1999), no. 1-2, 109-184.

[14] Generalized motion by nonlocal curvature in the plane, Arch. Ration. Mech. Anal. 159 (2001), no. 4, 295-333.

[15] M.-H. Giga, Y. Giga, and P. Rybka, A comparison principle for singular diffusion equations with spatially inhomogeneous driving force for graphs, Hokkaido University Preprint Series in Mathematics \#981 (2011).

[16] Y. Giga, Surface evolution equations: a level set approach, Birkhäuser Verlag, Basel, 2006.

[17] Y. Giga and P. Rybka, Facet bending in the driven crystalline curvature flow in the plane, J. Geom. Anal. 18 (2008), no. 1, 109-147.

[18] H. Ishii, Perron's method for Hamilton-Jacobi equations, Duke Math. J. 55 (1987), no. 2, 369-384. 
[19] J. E. Taylor, Constructions and conjectures in crystalline nondifferential geometry, Differential geometry, Pitman Monogr. Surveys Pure Appl. Math., vol. 52, Longman Sci. Tech., Harlow, 1991, pp. 321-336.

[20] _ Motion of curves by crystalline curvature, including triple junctions and boundary points, Differential geometry: partial differential equations on manifolds (Los Angeles, CA, 1990), Proc. Sympos. Pure Math., vol. 54, Amer. Math. Soc., Providence, RI, 1993, pp. 417438.

Graduate School of Mathematical Science, University of Tokyo, Komaba 3-8-1, MEguro-Ku, TOKYO 153-8914, JAPAN

E-mail address: mihogiga@ms.u-tokyo.ac.jp

Graduate School of Mathematical Science, University of Tokyo, Komaba 3-8-1, Meguro-Ku, TOKYO 153-8914, JAPAN

E-mail address: labgiga@ms.u-tokyo.ac.jp

Graduate School of Mathematical Science, University of Tokyo, Komaba 3-8-1, Meguro-Ku, TOKYO 153-8914, JAPAN

E-mail address: ankys@ms.u-tokyo.ac.jp 